\section{Intersections}

Canadian Journal of Music

Revue canadienne de musique
Intersections

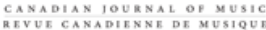

\title{
Composer en tant que catholique : une relecture de la musique vocale de Lili Boulanger
}

\section{Annegret Fauser et Marie-Hélène Benoit-Otis}

Volume 26, numéro 1, 2005

URI : https://id.erudit.org/iderudit/1013245ar

DOI : https://doi.org/10.7202/1013245ar

Aller au sommaire du numéro

Éditeur(s)

Canadian University Music Society / Société de musique des universités canadiennes

ISSN

1911-0146 (imprimé)

1918-512X (numérique)

Découvrir la revue

Citer cet article

Fauser, A. \& Benoit-Otis, M.-H. (2005). Composer en tant que catholique : une relecture de la musique vocale de Lili Boulanger. Intersections, 26(1), 113-122. https://doi.org/10.7202/1013245ar
Résumé de l'article

Par la mise en musique de textes des psaumes, Lili Boulanger s'est retrouvée partie prenante du débat sur le catholicisme dans la composition musicale qui faisait rage dans la France de la fin de siècle. Son choix de traduction des psaumes et son style musical révèlent un sens de l'archaïsme et de l'historicité en rapport avec le modernisme catholique, un mouvement en porte-à-faux avec les tendances néo-scolastiques de l'ultramontanisme romain des années précédant la Première Guerre Mondiale.
Copyright ( C Canadian University Music Society / Société de musique des universités canadiennes, 2006
Ce document est protégé par la loi sur le droit d'auteur. L'utilisation des services d'Érudit (y compris la reproduction) est assujettie à sa politique d'utilisation que vous pouvez consulter en ligne.

https://apropos.erudit.org/fr/usagers/politique-dutilisation/ 


\title{
COMPOSER EN TANT QUE CATHOLIQUE : UNE RELECTURE DE LA MUSIQUE VOCALE DE LILI BOULANGER
}

\author{
Annegret Fauser \\ Traduit de l'anglais par Marie-Hélène Benoit-Otis
}

En 1990, je me suis mise à la recherche de la musique perdue de Lili Boulanger, en quête de l'œuvre qu'avait occultée la légende de son auteur. Depuis ce temps, jai toujours concentré mon attention sur Boulanger la compositrice de musique moderne, contemporaine de Milhaud, Poulenc et Honegger (Fauser $1990)^{1}$. Mon travail avait pour objectif de déconstruire l'image publique de la femme fragile et de l'enfant prodige, qui semble avoir dominé la littérature sur Boulanger aussi bien durant sa vie que plusieurs décennies après sa mort. Je trouvais également que trop d'auteurs superposaient aux convictions religieuses de Boulanger les images de la vierge dévote et de lenfant souffreteux, trouvant la consolation dans la foi et la musique (voir en particulier Mauclair 1921 et Trieu-Colleney 1972). Trop souvent, l'imagination et l'ascendant de la musique de Boulanger étaient attribués à ses qualités angéliques, et sa mort prématurée était considérée comme la justification de cette inspiration divine - quand elle n’en devenait pas le prix à payer. Comment expliquer autrement l'accomplissement créatif et le travail innovateur d'une femme (Barraud 1968)? Compte tenu de cette rhétorique, il est compréhensible que j’aie emprunté une direction opposée en ignorant le catholicisme de Boulanger, à la faveur d'une vision plus séculière, moins sacro-sainte de la compositrice (Fauser 1997; 1998). Mais en mattardant au contexte social et artistique dans lequel a évolué Boulanger, du Prix de Rome à la poésie symboliste, et en ignorant le rôle important de sa foi catholique, j’ai omis une partie significative de son histoire personnelle. Le présent article constitue une première tentative pour corriger ce déséquilibre; jyy explorerai brièvement le rôle qu'a pu jouer la foi catholique dans le travail d'une compositrice du début du XXe siècle.

On sait très peu de chose sur la foi de Boulanger. Miki Piré, son amie et confidente, affirmait que Boulanger et elle " n’avaient jamais vraiment discuté de religion $^{2}$ ». Dans ses journaux intimes, Boulanger évoque des rencontres avec des hommes d'église, en particulier le Père Arthur Desprez, et mentionne parfois des prières ou des sujets de conversation; ainsi, en date du 2 juin 1916, elle note

1 Chailley 1982 constitue une exception digne dêtre mentionnée. Cet auteur souligne que pour comprendre l'œuvre de Boulanger, il importe de la restituer dans le contexte de la production de ses contemporains nés entre 1890 et 1895, notamment Honegger, Milhaud, Ibert et Hindemith (Chailley 1982, 19).

2 « Had never really discussed religion together » (Rosenstiel 1978, 133). 
dans son journal quelle a discuté de théosophie avec le Père Arthur ${ }^{3}$. Assister à la messe était bien sûr très important aussi bien pour Boulanger que pour sa famille, et selon Nadia Boulanger, léducation catholique a représenté une part essentielle de la formation spirituelle et morale des deux sœurs (Monsaingeon 1985,82 ). Un document remontant à l'enfance de Lili Boulanger évoque cette dernière, à huit ans, interprétant une messe musicale à Notre-Dame de Trouville avec sa sœur Nadia et Juliette Toutain (Rosenstiel 1978, 36). Plus tard, en 1916, une photographie montre la famille Boulanger, Lili tout en blanc, quittant une audience auprès du pape Benoît $\mathrm{V}$. Cette photo témoigne sans doute de la réputation de Boulanger, car toute jeune fille de 22 ans nobtient pas facilement une audience auprès du Saint Père. Enfin, l'annonce des funérailles de la compositrice précise qu'elle est morte "fortifiée par les sacrements de l'Église ${ }^{4}$ ".

Ces bribes d'informations permettent de conclure que pour Lili Boulanger, la religion représentait à la fois une affaire personnelle et un mode de vie. En apparence, elle semble avoir été une jeune fille rangée, dont les écrivains édifiants de la fin du XIX ${ }^{\mathrm{e}}$ siècle comme Monseigneur Dupanloup ou Clarisse Bader auraient approuvé la bonne éducation bourgeoise et catholique ${ }^{5}$. Que sa production s'achève en 1918 sur un Pie Jesu, une œuvre qu'elle a dictée à Nadia parce que sa faible main était incapable de tenir un crayon, pourrait sembler soutenir cette thèse, si ce nétait du caractère frondeur de cette œuvre dont l'intertexte comprend une autre réalisation musicale des mêmes mots, beaucoup plus réconfortante, réalisée par l'ami de la famille Gabriel Fauré. Contrairement à son aîné, Boulanger n'exprime pas la consolation, mais emprunte plutôt un langage troublant et polytonal, qui laisse en suspens la question de savoir si la musique conduira au repos éternel - requiem eternam - comme le demande le texte (Mattis 1993). Cette réalisation musicale d'un texte liturgique, présumément destinée à un usage privé, pourrait même ouvrir la voie à une nouvelle interprétation de l'histoire traditionnelle de Boulanger. À la place de l'adolescente dévote et frêle, l'œuvre révèle une personnalité forte qui se pose des questions et n'accepte pas sans esprit critique les vérités communément admises. En fait, cette hypothèse nest point surprenante.

La famille Boulanger entretenait des liens avec de nombreux intellectuels et artistes de tous horizons, de l'architecte William Bouwens van der Boijen et de larchéologue Théophile Homolle aux poètes Gabriele D’Annunzio et Emile Verhaeren, en passant par l'orientaliste Suzanne Karpelès, et une amie de Rabindranath Tagore, Louise Gonet (Rosenstiel 1978, 33-34). Les journaux intimes de Boulanger font souvent référence à des dîners agrémentés de discussions animées, malheureusement sans préciser les sujets de conversation. On sait cependant par Nadia Boulanger que ces conversations, au cours desquelles Lili ne cédait pas sa place, étaient vives, stimulantes, et abordaient une grande variété de sujets (Rosenstiel 1978, 34). Ainsi, l'expérience de Boulanger semble refléter

3 BNF-Département de la Musique, Fonds Boulanger, Agenda 1916, entrée du 2 juin 1916 : « Je reste avec $P$. Arthur, avec qui parlons théosophie etc. ".

4 "Fortified with the rites of the Church " (Rosenstiel 1978, 133).

5 Voir, par exemple, Bader 1883; Dupanloup 1879. Martin-Fugier 1983 a montré jusqu’à quel point ces idées étaient encore présentes dans léducation des femmes au tournant du $\mathrm{XX}^{\mathrm{e}}$ siècle. 
celle d'autres intellectuels et artistes parisiens - de Fauré à Jammes et, plus tard, Messiaen - en ceci que son éducation et sa pratique religieuses de catholique convaincue lui servaient de point d'ancrage pour explorer, juger et éventuellement s'approprier d'autres croyances et idées.

Cette forme de catholicisme intégrateur plutôt qu'exclusif représente un courant parmi d'autres du catholicisme français de la fin du XIX et du début du $\mathrm{XX}^{\mathrm{e}}$ siècles. La période de 1890 à 1914 ne se réduit pas uniquement à l'antagonisme bien connu entre l'Église et l'État, qui va conduire aux réformes anticléricales, et à la séparation formelle de 1905. Elle est également le théâtre d'un débat non moins acrimonieux au sein même de l'Église catholique, opposant les modernistes à la suite d'Alfred Loisy et de Maurice Blondel, aux anti-modernistes scolastiques inspirés par Pie $\mathrm{X}$, le pape réactionnaire nouvellement élu' 6 . Les modernistes catholiques cherchent à établir une théologie historiquement responsable, aux antipodes de la perpétuation du dogme ecclésiastique ultramontain visée par l'encyclique Pascendi de Pie X (1906). Cette dernière ne se contente pas d'accroître l'autorité du pape, elle s'oppose en plus à létude historique de la Bible, soutient la néo-scolastique, et de façon générale, tente de freiner toute érosion de l'influence de l'Église (Jodock 2000, 25-26). En France, la nouvelle forme de piété conservatrice ultramontaine, fortement encouragée par Rome, s'exprime surtout dans la dévotion à Marie (en particulier dans le cas de Lourdes), la diffusion de l'adoration eucharistique dans le culte du Sacré-Cœur et l'anti-intellectualisme de l'enseignement religieux (Gibson 1989, 265).

Sur la base des documents disponibles, je crois pouvoir affirmer quen ce qui concerne la théologie, Boulanger était portée vers le modernisme catholique. Sa pratique religieuse était bien éloignée de la dévotion à la Vierge et au Sacré-Cœur associée au catholicisme anti-moderne, sans parler des pèlerinages à Lourdes. Au contraire, elle semble avoir fait preuve douverture aux autres confessions et religions, en raison notamment de son entourage partiellement russe (plusieurs de ses amis et de ses domestiques pratiquaient la religion russe orthodoxe). De plus, il est fort possible quelle se soit intéressée à la théosophie; c'est du moins ce que semble indiquer sa réalisation musicale de la Vieille prière bouddhique, dont le contenu associé à la philosophie indienne fait écho à des préoccupations théosophiques ${ }^{7}$. Cette ouverture à la théosophie est également reflétée par la fascination de Boulanger pour la symbolique des nombres (Dopp 1994), ainsi que par son usage d'un psautier traduit par Henri Lesêtre, qui semble avoir été lié, au moins de façon périphérique, au courant du modernisme catholique avant d'adhérer plus tard à la fraternité théosophique des " Polaires ${ }^{8}$ ".

La musique de Boulanger, et plus particulièrement ses œuvres vocales, semble étayer la thèse d'une pratique catholique modérée. Il est révélateur que sa production religieuse mette surtout en musique des textes de psaumes (voir le tableau ci-dessous). Dans la liturgie catholique, les psaumes sont chantés à l'of-

6 Pour une brève reconstitution des préoccupations catholiques au tournant du $\mathrm{XX}^{\mathrm{e}}$ siècle, voir Duroselle 1992; sur Loisy, voir Talar 2000.

7 La Vieille prière bouddhique a fait l'objet d'une thèse inédite : Ruhbaum 1994.

8 La Fondation Internationale Nadia et Lili Boulanger détient une copie de Lesêtre, 1913. Je suis reconnaissante à Alexandra Laederich de mavoir fourni cette information. 
fice, et non durant la messe ${ }^{9}$. De plus, depuis le XVIe siècle, les psaumes sont associés en France à l'individualité de la piété protestante plutôt quà la pratique plus collective du catholicisme. Un bref survol de l'œuvre des prédécesseurs et des contemporains de Boulanger montre que la réalisation musicale de psaumes constitue une partie négligeable de leur production, même dans le cas de compositeurs de musique d’église aussi réputés que Widor (qui a mis en musique deux psaumes) et Vierne (qui n'en a écrit aucun) ${ }^{10}$. Mais tous ont composé des messes, des Ave Maria, ou d'autres textes liturgiques s'inscrivant soit dans le contexte de la messe, soit dans celui de la piété personnelle de la France des $\mathrm{XIX}^{\mathrm{e}}$ et $\mathrm{XX}^{\mathrm{e}}$ siècles.

CEuvres DE CARACTÈre SACrÉ DE LILI BoulaNGer

\begin{tabular}{|c|c|c|}
\hline Psaumes & $\begin{array}{l}\text { Autres Cuvres } \\
\text { liturgiques }\end{array}$ & Autres \\
\hline \multicolumn{3}{|c|}{ CEuvres conservées } \\
\hline $\begin{array}{l}\text { Ps 129, «Ils mont assez } \\
\text { opprimé », 1910-16 }\end{array}$ & Pie Jesu, 1918 & $\begin{array}{l}\text { Pour les funérailles d'un soldat (A. de } \\
\text { Musset), 1912-13 }\end{array}$ \\
\hline $\begin{array}{l}\text { Ps 130, «Du fond de } \\
\text { labîme », 1910-17 }\end{array}$ & & $\begin{array}{l}\text { Vieille prière bouddhique (extrait du } \\
\text { Visuddhimagga, traduit par S. Kar- } \\
\text { pelès), 1914-17 }\end{array}$ \\
\hline $\begin{array}{l}\text { Ps } 24, \text { « La terre appartient à } \\
\text { l'Eternel », } 1916\end{array}$ & & \\
\hline \multicolumn{3}{|c|}{ Euvres perdues } \\
\hline Ps 131,1907 & Ave Maria, 1908 & Apocalypse, 1909 \\
\hline Ps 137,1907 & & 1 Corinthiens 13,1909 \\
\hline \multicolumn{3}{|l|}{ Ps 1,1909} \\
\hline Ps 119,1909 & & \\
\hline
\end{tabular}

La musique religieuse vocale de Boulanger semble donc se démarquer de celle de la France de son époque par le choix de ses textes. Sur le plan textuel, les psaumes offrent au compositeur plusieurs avantages : ils sont religieux sans faire nécessairement partie de la liturgie; ils proviennent d'un corpus de textes archaïques, d'un caractère " primitif " en comparaison avec la littérature chrétienne plus récente; lemploi de la première personne (le « je " lyrique du Roi David) leur confère un caractère individuel; et enfin, ils détiennent l'autorité qui revient à tout texte biblique. En fait, peu de textes expriment aussi fortement les aspirations d'une compositrice qui, selon l'aveu de son journal intime,

9 Sur le psaume dans la liturgie catholique, voir Harper 1991, 67-72. Le chapitre suivant (73-108) contient des propos très détaillés sur looffice.

10 Une brève enquête préliminaire a donné les résultats suivants : Vincent d'Indy, Camille SaintSaëns, Florent Schmitt, Charles Tournemire et Charles-Marie Widor ont mis en musique chacun deux psaumes; César Franck, Gabriel Fauré et Albert Roussel chacun un; Louis Aubert, Charles Bordes, Nadia Boulanger, Alfred Bruneau, Gustave Charpentier, Claude Debussy, Maurice Delage, Augusta Holmès, Charles Koechlin, Jules Massenet, Francis Poulenc, Maurice Ravel, Jean Roger-Ducasse et Louis Vierne aucun. Aucun des compositeurs considérés dans cette étude na écrit plus de deux psaumes. 
conçoit la musique comme un acte humanitaire et politique ${ }^{11}$. En quête d'un sujet dopéra en 1915, Lili Boulanger cherche un livret qui aborde le thème de la guerre ${ }^{12}$. Avec Pour les funérailles d'un soldat et la Vieille prière bouddhique, les trois psaumes mis en musique par Boulanger et qui ont été conservés forment un corpus portant sur des sujets humanistes, et que l'on peut lire dans le contexte de la Première guerre mondiale. Le Psaume 24, en particulier, rappelle à ses auditeurs que " la terre appartient au Seigneur » et que c'est Lui qui possède les armées. Le Psaume 24 a été conçu en 1915 et terminé en 1916.
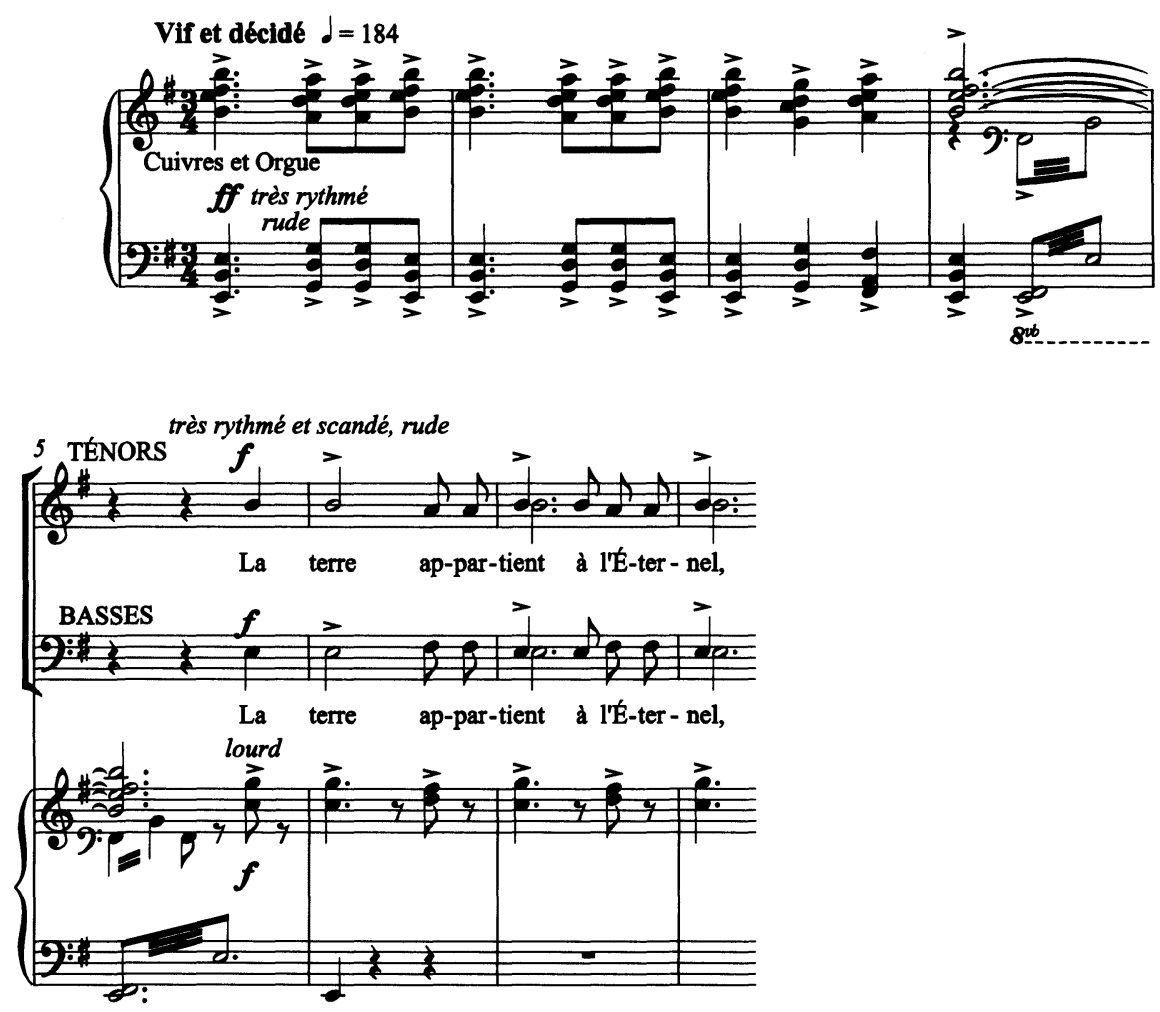

(8)

Exemple 1 : Lili Boulanger, Psaume 24 (mes. 1-8)

La réalisation musicale du Psaume 24 par Boulanger peut aider à expliquer pourquoi ses œuvres chorales de grande envergure dans les années 1910 utilisent des psaumes. Cette musique rappelle une œuvre qui a pu constituer une influence déterminante sur sa découverte des psaumes, le Psaume 47, opus 38 de Florent Schmitt. Lorsque le Psaume de Schmitt est créé en France le 27 décem-

11 Voir Fauser 1997, 75. Voir aussi la description que fait Eduard Reeser d'un poème symphonique perdu, dans lequel il trouve des impressions de paysages remplis du « son des canons, de la nuit, des morts, de la douleur et de la solitude " (" die de plat geschoten vlakten, de nacht, de gewonden, de smart en de eenzaamheid oproepen ", Reeser 1932/33, 269).

12 Lettre de Paul Gentien (Ricordi) à Lili Boulanger, 2 septembre 1915(?), Bibliothèque nationale de France, Département de la Musique, Fonds Boulanger. 
bre 1906, Nadia Boulanger tient la partie d'orgue. Selon cette dernière, sa sœur est fascinée par l'œuvre de Schmitt, et n'en manque pas une seule répétition (Rosenstiel 1978, 47). Et en effet, la réalisation musicale du Psaume 24 par Boulanger peut être considérée comme un intertexte du Psaume de Schmitt, aussi bien sur le plan musical que littéraire.
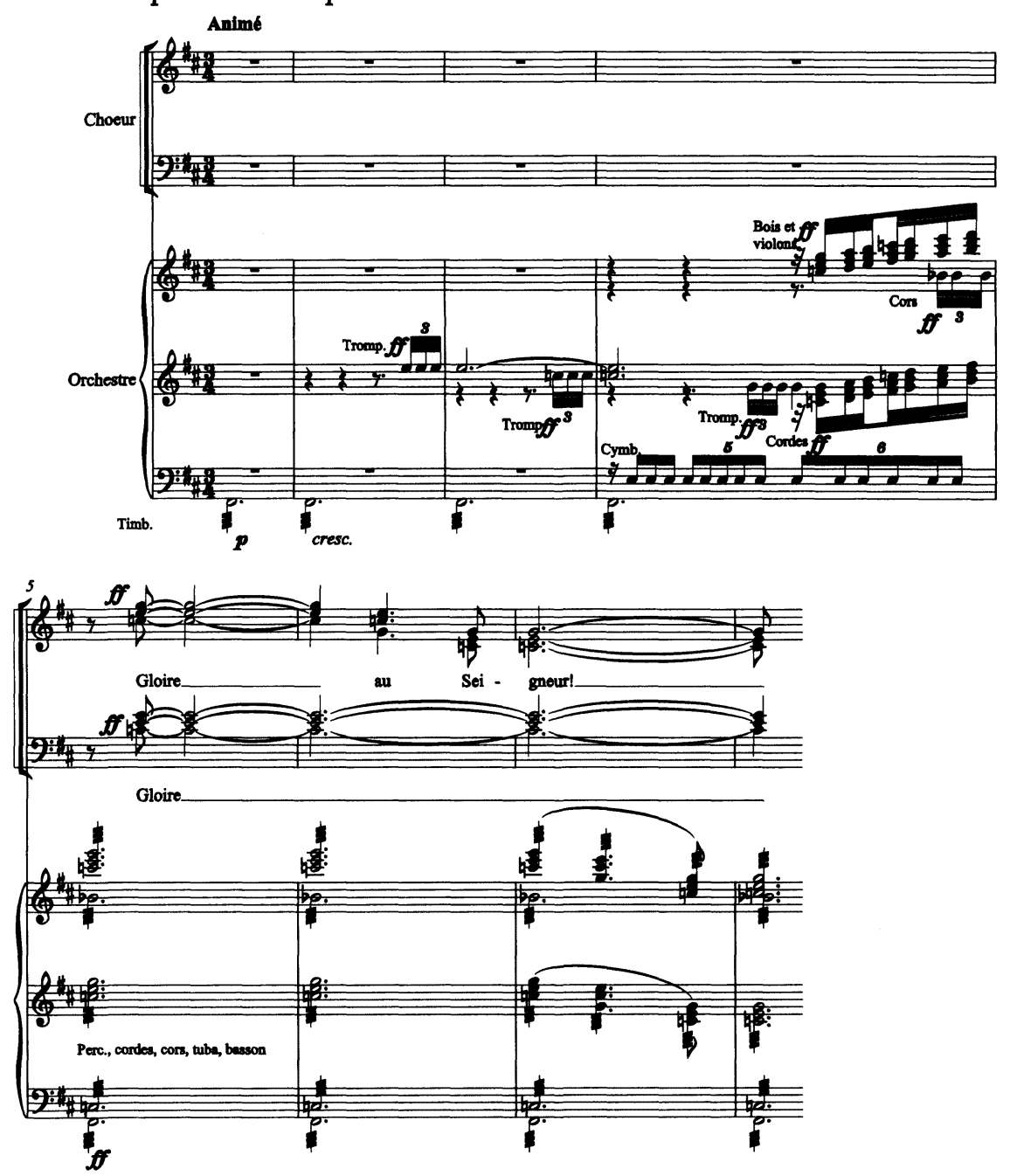

Exemple 2: Florent Schmitt, Psaume 47 (mes. 1-8)

Les similarités entre louverture du Psaume 24 de Boulanger et celle du Psaume $47 \mathrm{de}$ Schmitt sont frappantes. Même la structure musicale des deux œuvres est similaire : l'une comme l'autre commencent par une section chorale homophonique, suivie par une partie centrale pour un soliste, et se terminent par une seconde section chorale. Le geste rythmique initial de Boulanger (joué par les cuivres) est remarquablement similaire à celui de Schmitt, tout comme la prosodie de la section chorale douverture. 
L'œuvre de Schmitt jouit d'une grande notoriété dans la première partie du $\mathrm{XX}^{e}$ siècle et suscite l'émulation. Au début de l'année 1907, Boulanger commence à rédiger des esquisses pour ses tout premiers Psaumes (Psaumes 131 et 137, perdus) immédiatement après l'avoir entendue, et elle n'est pas la seule dans cette situation. Pour sa part, Charles Tournemire entame la composition de son Psaume 57, pour chœur et orchestre, en 1908. Plus tard, Messiaen reconnait l'influence du Psaume de Schmitt sur ses Trois petites liturgies (Vignal, 1989, 4). Il semble donc très probable que Schmitt ait donné à Boulanger l'impulsion initiale vers l'usage de psaumes pour sa musique vocale. Sur le plan de la réalisation musicale, la relation intertextuelle du Psaume 24 avec le Psaume $47 \mathrm{de} \mathrm{Schmitt}$ comporte d'autres résonances. Le Psaume 47 célèbre le pouvoir et la gloire de Dieu, tandis que le Psaume 24, beaucoup plus sombre, cite l'"Eternel, fort et puissant dans la bataille ", faisant manifestement allusion à la guerre qui faisait rage au moment de la composition.

Mais le Psaume de Boulanger se distingue surtout par sa qualité "primitive " et volontairement archaïque, quon observe aussi bien dans la traduction du psaume que du langage musical lui-même. Cela s'applique d'ailleurs également à ses deux autres Psaumes. Ainsi Boulanger choisissait la traduction de Lesêtre du Psaume 129, "Du fond de l'abîme ", qui utilise les appellations de "Iahvé » et " Adonaï " plutôt que "Seigneur ", rendant plus archaïque la sonorité du texte (Lesêtre 1913, 221-22) ${ }^{13}$. Ce psaume, dont le lien avec Le sacre du printemps et d'autres œuvres de Stravinsky a souvent été observé, s'inscrit plus fortement dans un modernisme musical que le Psaume 24. Les journaux intimes de Boulanger révèlent qu'elle étudie la musique de Stravinsky en 1916 au moment même où elle travaille à un des psaumes (probablement 129) : "Vais au piano - travaille un peu au psaume - que je reprends - puis reste couchée - vais après déj. au piano - prends le Rossignol, de Stravinsky ${ }^{14}$ ". Son journal de l'époque révèle également une préoccupation pour les modes et pour le contrepoint. En novembre 1916, par exemple, elle note qu'une discussion avec Nadia sur les modes anciens - " chantons toutes deux contrepoints improvisés ${ }^{15}$ ". Quelques mois plus tôt, pour passer le temps, elle avait composé une fugue simplement pour voir ce qu'il était possible de faire avec un sujet ${ }^{16}$. Le Psaume 24 , comme les deux autres Psaumes, contient de longs passages modaux, et dans le Psaume 24, un diatonisme contrastant colore la partie de ténor solo au moment où le texte aborde la bénédiction de ceux qui ont les mains propres et ne commettent pas de parjure. Le caractère archaïque du langage tonal au début de l'œuvre est également soutenu par lorchestration (qui fait appel aux cuivres, la harpe, lorgue et les timbales), et par la première indication d'exécution pour le chœur, " très rythmé et scandé, rude " (Boulanger 1924, 1). Elle utilise un contraste musical

13 Bien que Boulanger utilise la traduction de Lesêtre comme texte principal, elle y ajoute des exclamations et des variantes.

14 BNF-Département de la Musique, Fonds Boulanger, Agenda 1916, entrée du 27 mai 1916.

15 BNF-Département de la Musique, Fonds Boulanger, Agenda 1916, entrée du 20 novembre 1916. Le 20 décembre, elle note : "Nadia me fait improviser. "

16 BNF-Département de la Musique, Fonds Boulanger, Agenda 1916, entrée du 25 mai 1916 : "Fais de la fugue, au lit - sur le sujet de Fauré, de l'examen, afin de voir ce qu’on en pouvait tirer. " 
même plus prononcé dans son Psaume 129 pour exprimer la différence entre le désespoir initial et l'invocation à la clémence et à la délivrance de la seconde partie, bien quà la fin, elle retourne au texte et aux sonorités du début.

Les trois Psaumes de Boulanger sont caractérisés par un jeu entre idiomes modernistes, archaïsmes modaux, un certain primitivisme rythmique et des moments de beauté nostalgique. L'archaïsme est également typique du choix de textes de Boulanger, aussi bien pour ces Psaumes que pour la Vieille prière bouddhique. Sa musique et ses textes font allusion aux origines d'une façon similaire à celle d'autres compositeurs contemporains à la recherche de langages originaux et archaïques, dans le but d'atteindre une forme d'expression artistique plus vraie et plus directe : on pense, par exemple, à La création du monde de Milhaud ou à la Symphonie des psaumes de Stravinsky. Les Psaumes de Boulanger s'adressent à l'humanité et critiquent la destruction mutuelle des êtres humains dans des actes de barbarie guerrière. Ainsi, pour Lili Boulanger, composer en tant que catholique pendant la Première guerre mondiale n'est pas une façon de se retirer dans le monde privé de la musique dévotionnelle : cela signifie plutôt la création d'œuvres de grande envergure, s'adressant à un vaste auditoire, et non uniquement à Dieu.

\section{RÉFÉRENCES}

Bader, Clarisse. 1883. La femme française dans les temps modernes. Paris: E. Perrin.

Barraud, Henri. 1968. "Regards sur la musique». Reproduit dans Birgit Stièvenard-Salomon 1993, 53.

Boulanger, Lili. 1924. Psaume XXIV, transcription pour chant et piano par lauteur. Paris : Durand \& Cie.

Chailley, Jacques. 1982. "L'œuvre de Lili Boulanger». La Revue musicale, $\mathrm{n}^{0}$ 353-354, numéro spécial Lili et Nadia Boulanger : 15-44.

Dopp, Bonnie Jo. 1994. « Numerology and Cryptography in the Music of Lili Boulanger: The Hidden Program in Clairières dans le ciel». The Musical Quarterly 78, $\mathrm{n}^{\circ}$ 3: 557-583.

Mgr Dupanloup, évêque d'Orléans. 1879. Lettres sur léducation des filles et sur les études qui conviennent aux femmes dans le monde, seconde édition. Paris : J. Gervais.

Duroselle, Jean-Baptiste. 1992. La France de la "Belle Époque ». Paris, Presses de la Fondation Nationale des Sciences Politiques. Réimpression de l'édition de 1972.

Fauser, Annegret. 1990. « Die Musik hinter der Legende. Lili Boulangers Liederzyklus Clairières dans le ciel ». Neue Zeitschrift für Musik 151, n 11 (novembre) : 9-14.

1997. "Lili Boulanger's La Princesse Maleine: A Composer and Her Heroine as Literary Icons ». Journal of the Royal Musical Association 122, $\mathrm{n}^{0} 1: 68-108$. 
1998. "La guerre en dentelles: Women and the Prix de Rome in French Cultural Politics ". Journal of the American Musicological Society $51, \mathrm{n}^{\circ} 1: 83-129$.

Gibson, Ralph. 1989. A Social History of French Catholicism, 1789-1914. London and New York : Routledge.

Harper, John. 1991. The Forms and Orders of Western Liturgy from the Tenth to the Eighteenth Century: A Historical Introduction and Guide for Students and Musicians. Oxford : Clarendon Press.

Jodock, Darrell, dir. 2000. Catholicism Contending with Modernism: Roman Catholic Modernism and Anti-Modernism in Historical Context. Cambridge : Cambridge University Press.

Lesêtre, Henri, curé de Saint-Étienne du Mont. 1913. Les psaumes du bréviaire, traduits de l'hébreu. Paris : P. Lethielleux.

Mattis, Olivia. 1993. « Lili Boulanger - Polytoniste ». Lili Boulanger Tage 1993. Bremen, zum 100. Geburtstag der Komponistin, sous la dir. de Kathrin Mosler, 48-50. Bremen : CALLAS.

Mauclair, Camille. 1921. "La vie et l'œuvre de Lili Boulanger ». La Revue musicale, $\mathrm{n}^{\circ} 10: 147-155$.

Martin-Fugier, Anne. 1983. La bourgeoise: femme au temps de Paul Bourget. Paris : Bernard Grasset.

Monsaingeon, Bruno. 1985. Mademoiselle: Conversations with Nadia Boulanger. Trad. par Robyn Marsack. Manchester : Carcanet..

Reeser, Eduard. 1932/33. "Lili Boulanger ». De Muziek, 7, n 5/6: 210-21, 264-71.

Rosenstiel, Leonie. 1978. The Life and Works of Lili Boulanger. Rutherford, Madison, and Teaneck : Fairleigh Dickinson University Press.

Ruhbaum, Antje. 1994. Das "Vieille prière bouddhique" von Lili Boulanger: Ein Beispiel für den Einfluß außereuropäischer Kulturen auf die französische $\mathrm{Mu}$ sik der Jahrhundertwende. Hausarbeit zur künstlerisch-wissenschaftlichen Staatsprüfung, Berlin, Hochschule der Künste.

Stièvenard-Salomon, Birgit. 1993. Lili Boulanger : l'œuvre retrouvée. Issy-lesMoulineaux : Municipalité d'Issy-les-Moulineaux.

Talar, C.J.T. 2000. "Innovation and biblical interpretation ». Dans Catholicism Contending with Modernism : Roman Catholic Modernism and Anti-Modernism in Historical Context, sous la dir. de Darrell Jodock, 191-211. Cambridge : Cambridge University Press.

Trieu-Colleney, Christiane. 1972. «Lili Boulanger (1893-1918) ou Musique pour une éternité ". Jeunesse et Orgue, Reproduit dans Birgit StièvenardSalomon 1993, 88.

Vignal, Marc. 1989. Notes de programme pour l'enregistrement des Trois petites liturgies de Messiaen. Paris : Erato. 


\section{ABSTRACT}

Lili Boulanger took part musically in the lively debates in fin de siècle France about Catholicism through her compositions, in particular her psalm settings. Her choice of psalm translation and her musical style reveal a sense of archaism and historicity that relates to catholic modernism, a movement at odds with the neo-scholastic trends of Roman ultramontanism in the years before World War I.

\section{RÉSUMÉ}

Par la mise en musique de textes des psaumes, Lili Boulanger sest retrouvée partie prenante du débat sur le catholicisme dans la composition musicale qui faisait rage dans la France de la fin de siecle. Son choix de traduction des psaumes et son style musical révèlent un sens de l'archaïsme et de l'historicité en rapport avec le modernisme catholique, un mouvement en porte-à-faux avec les tendances néo-scolastiques de lultramontanisme romain des années précédant la Première Guerre Mondiale. 